\title{
Opinions of dentists and directors of nursing concerning dental care provision for Adelaide nursing homes
}

\author{
JM Chalmers," C Hodge, $†$ JM Fuss," AJ Spencer,* KD Carter," R Mathew*
}

\begin{abstract}
Background: The complex oral health problems of nursing home residents have been well documented. However, the influences on residents' oral health status, including opinions and experiences of dental professionals and nursing home staff, have not yet been adequately investigated.

Methods: The baseline questionnaire component of this longitudinal study was mailed to all registered dentists practising in Adelaide and Adelaide nursing home directors of nursing (DONs).

Results: 413 dentists and 97 DONs indicated that Adelaide dentists' interest and training in nursing home dentistry was low. Dental service provision for nursing home residents was very low and dentists preferred to provide treatment at their dental practices. Few dental hygienists were working in nursing homes and dental professionals provided little educational assistance for nursing home staff. Dentists and DONs held several common and many varying perceptions of the problems associated with dental care provision in nursing homes. Both identified a group of nursing home environmental constraints and a lack of portable dental equipment. DONs further identified a group of resident related problems, and dentists a group of dental practicerelated problems.

Conclusions: These study results provide important information concerning problems with nursing home dentistry for dental service providers, educators, policy-makers, administrators and nursing home staff.
\end{abstract}

Key words: Nursing homes, older adults, dental care provision, directors of nursing, dentists

(Received for publication February 2000. Revised May 2000. Accepted August 2000.)

\section{INTRODUCTION}

The complex oral health problems of functionally dependent older adults residing in nursing homes have been well documented in Australia and other industrialised countries. ${ }^{1-9}$ However, the many influences on residents' oral health status have not yet been

\footnotetext{
*AIHW Dental Statistics and Research Unit, The University of Adelaide.
}

†Australian Dental Association, South Australian Branch. adequately investigated. In North America, several studies have investigated the opinions and experiences of dental professionals and nursing home staff concerning residents' oral health problems and dental care provision at nursing homes. ${ }^{10-17}$ Dental professionals and nursing staff were found to have some common but also many differing perceptions of the influences on the provision of residents' dental treatment and oral hygiene care. ${ }^{11,15,17}$ Questionnaires to dentists have found that dental service provision at nursing homes was constrained by a lack of portable equipment, difficult working conditions at nursing homes, dentists' private practice pressures, and dentists' concerns about inadequate training. ${ }^{10-13}$ Such information is essential for planning appropriate and effective strategies to improve the oral health of nursing home residents. However, no similar studies have been conducted with Australian dental professionals and nursing home staff.

The Adelaide Dental Study of Nursing Homes was a longitudinal study instigated by the Australian Dental Association (ADA) (South Australian Branch) and the AIHW Dental Statistics and Research Unit in 1998. There were two components in the baseline data collection for the study. ${ }^{9}$ Clinical dental inspections were completed for residents of randomly selected Adelaide nursing homes. Opinions and experiences of Adelaide dental professionals and nursing home staff were investigated using questionnaires mailed to all practising Adelaide dentists and Adelaide nursing home directors of nursing (DONs). This paper will present the results of the questionnaire component of the baseline data collection. The aims of the questionnaire component were to:

1) quantify the dental care provided for Adelaide nursing home residents by Adelaide dental professionals in the 12 months prior to baseline data collection;

2) investigate the attitudes of Adelaide dentists and DONs toward dental care for nursing home residents; and

3) identify the problems most frequently encountered with the organisation and provision of dental care for residents of nursing homes, as reported by dentists and DONs. 


\section{METHODS}

Ethical approval for The Adelaide Dental Study of Nursing Homes was obtained from The University of Adelaide Human Ethics Committee. A pilot questionnaire was mailed in late 1997, to all South Australian country dentists registered with the ADA (SA Branch) Nursing Home Scheme and to the DONs of the SA country nursing homes listed with the Aged Care Division of the Commonwealth Department of Health and Family Services.

The final study questionnaire was mailed to Adelaide dentists and DONs in early 1998. A list of all practising Adelaide dentists, excluding registered specialists, was obtained from the Dental Board of South Australia. All 531 general dentists listed as currently practising were included in the questionnaire mailout. A list of all Adelaide nursing homes was obtained from the Aged Care Division of the Commonwealth Department of Health and Family Services, and questionnaires were mailed to the 'Director of Nursing' at each of the 114 listed nursing homes. Two weeks later, a reminder letter was sent to non-respondents, and three weeks after this a second reminder with a replacement questionnaire was mailed.

Both dentist and director of nursing questionnaires had the same basic structure and content. In addition to one qualitative open-ended question for comments, close-ended questions were asked to quantify:

- participants' age and sex, and dentists' number of years in practice and practice location;

- dental service provision for nursing home residents by dentists, dental hygienists and dental technicians;

- attitudes of dentists and DONs toward:

- residents' need for regular dental examinations

- dentists' interest in nursing home dentistry

- awareness of SA dental hygienist regulatory changes permitting hygienists to work unsupervised to a dentist's prescribed treatment plan in nursing homes ${ }^{18}$

- awareness of the ADA (SA Branch) Nursing Home Scheme

- dentists' training in geriatric dentistry (only asked of dentists); and

- problems encountered with the organisation and provision of dental care for nursing home residents.

The problems encountered with the organisation and provision of dental care for nursing home residents were investigated using a block of nineteen randomly ordered statements with a 5-point Likert scale response. The list of problems was developed from:

- a review of literature; ${ }^{11-14}$

- consultation with DONs and nursing home staff; and

- consultation with dentists with clinical and research experience in Geriatric Dentistry.

Maintenance of the participant database, epidemiological data collection and statistical analyses were conducted using SPSS for Windows (Versions 6.1 and 8.0). Univariate statistics were computed to describe
Table 1. Dentists' practice characteristics by sex (\%).

\begin{tabular}{lccr}
\hline & Total & Male & Female \\
Age group (years)* & $\mathrm{n}=400$ & $\mathrm{n}=321$ & $\mathrm{n}=79$ \\
$\leq 24$ & 1.3 & 1.3 & 1.3 \\
$25-44$ & 52.0 & 45.8 & 77.2 \\
$45-64$ & 42.5 & 48.0 & 20.2 \\
$65+$ & 4.2 & 4.9 & 1.3 \\
Practice type* & $\mathrm{n}=407$ & $\mathrm{n}=325$ & $\mathrm{n}=82$ \\
Private only & 74.2 & 79.4 & 53.7 \\
Public only & 12.5 & 8.3 & 29.3 \\
Public and private & 6.4 & 5.2 & 11.0 \\
Other & 6.9 & 7.1 & 6.0 \\
Years in practice* & $\mathrm{n}=402$ & $\mathrm{n}=321$ & $\mathrm{n}=81$ \\
1-5 & 9.5 & 6.9 & 19.7 \\
6-10 & 11.7 & 7.8 & 27.2 \\
11-20 & 35.6 & 34.9 & 38.3 \\
21-30 & 32.3 & 37.0 & 13.6 \\
$30+$ & 10.9 & 13.4 & 1.2 \\
\hline
\end{tabular}

"chi-square test $\mathrm{p}<0.01$

participants' demographic characteristics and their responses concerning residents' need for regular dental examinations, dentists' interest in nursing home dentistry, awareness of SA dental hygienist regulations, use of dental hygienists in nursing homes, and awareness of ADA (SA Branch) Nursing Home Scheme. Tests of significance (Pearson's chi-square statistic) were used to describe the differences among dentists' demographic characteristics and their practice characteristics, dental service provision for nursing homes, and awareness of the ADA (SA Branch) Nursing Home Scheme.

Univariate statistics and tests of significance ( $t$-test) were used to describe the problems encountered with the organisation and provision of dental care as reported by dentists and DONs. Logistic regression analysis was used to model characteristics of dentists who had provided dental care for residents at nursing homes during the previous 12 months.

\section{RESULTS}

Questionnaire response rates were high for both dentists $(n=413)$ (78 per cent) and DONs $(n=97)(85$ per cent). The majority of participating dentists were older males who had worked in private practice for $11+$ years (chi-square $\mathrm{p}<0.01$ ) (Table 1 ). Over 60 per cent of dentists indicated that they had not received adequate clinical training in nursing home dentistry, over 50 per cent had not received adequate undergraduate training in geriatric dentistry, and over 40 per cent had not received adequate training in clinical care of medically compromised older adults (Table 2). Nearly 50 per cent of dentists had provided dental care for residents of one to two nursing homes in the previous year. However, the quantity of care provided was small, especially at nursing homes (Table 2). Only 29.5 per cent of dentists had provided care at nursing homes, the majority spending less than two hours per month doing so. Dentists clearly preferred to treat residents off-site from nursing homes at their dental practices/clinics. Dental treatment was provided at off-site dental practices/clinics by more than 80 per cent of dentists, with 40 per cent providing care off-site only. The use of dental hygienists in nursing home dentistry was reported infrequently, 
Table 2. Dental care provision for Adelaide nursing homes (\% of dentists).

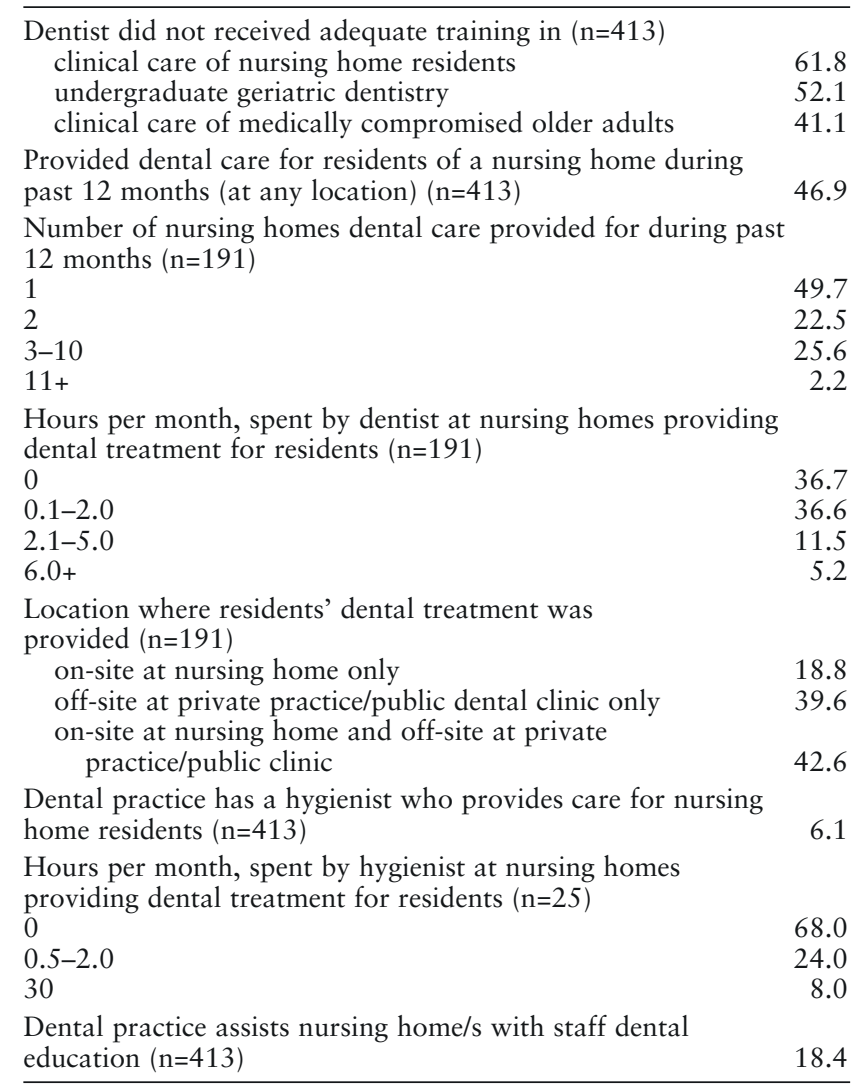

with only 6 per cent of practices utilising a hygienist at nursing homes. Less than 20 per cent of practices assisted nursing homes with staff education about residents' oral care (Table 2 ).

DONs were asked to identify the type of dental staff who had provided care for residents at their nursing home during the previous year (Figure 1). DONs reported the use of both the public domiciliary service (59 per cent) and private dentists (57 per cent). Approximately one-fifth of DONs reported that a dental technician had provided treatment for residents' dentures, and 12 per cent of DONs reported that a dental hygienist had treated residents at the nursing home.

Dentists' interest in nursing home dentistry was low (Table 3). More than half of the dentists were not interested or only somewhat interested in providing dental care for residents. Dentists' interest did not significantly differ by age, sex, or years in practice. DONs' also perceived dentists' interest to be low.

Dentists' and DONs' awareness of changes to the dental hygienist regulations in SA and of the ADA (SA Branch) Nursing Home Dental Scheme were both low (Table 3). Dentists' and DONs' awareness of the dental hygienist regulation change did not significantly differ by their age or sex. There were no significant differences among DONs' age groups or sex concerning their awareness of the ADA Nursing Home Scheme. As Figure 2 shows, dentists' awareness of the ADA scheme varied by age group. Older dentists were significantly more aware of the scheme than were younger dentists

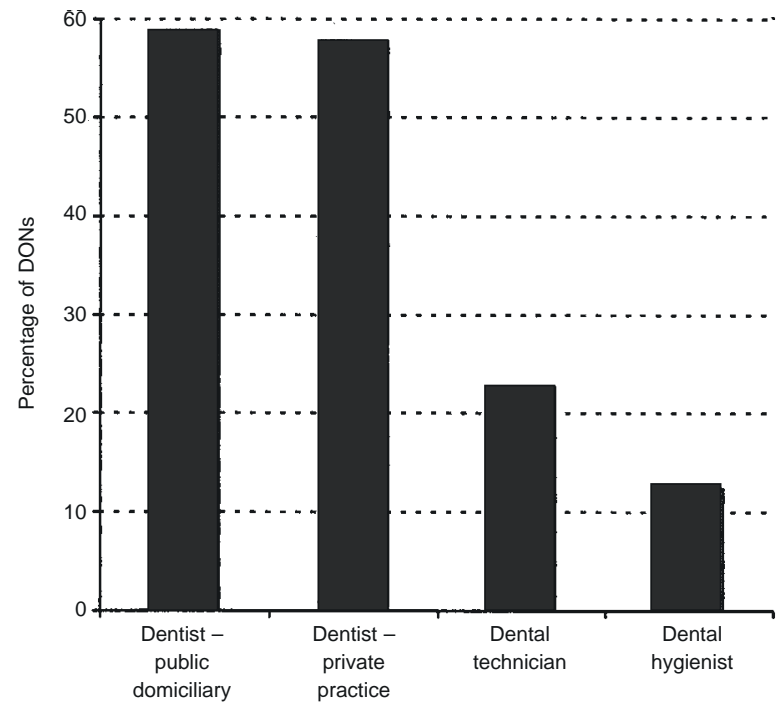

Fig. 1. Types of dental staff who provided care at nursing homes during the previous 12 months, as reported by DONs $(n=97)$.

(chi-square $\mathrm{p}<0.01$ ). The majority of dentists and DONs indicated that some form of regular dental examination by a dentist was required for dentate and edentulous residents. However, examinations were thought to be required less frequently for edentulous residents.

Dentists' characteristics were modelled using logistic regression to identify those who had provided care at nursing homes for residents during the previous year. Males, aware of the ADA Nursing Home Scheme, who were very or extremely interested in nursing home dentistry, were more likely to have provided care at nursing homes $(\mathrm{p}<0.05)$ (Table 4$)$.

Table 5 shows how dentists and DONs rated the frequency of the statements on problems they encountered with the organisation and provision of dental care for residents. As dentists consistently rated problems more frequently than did DONs, scores were standardised to the group mean (dentists and DONs) to

Table 3. Attitudes toward nursing home dentistry.

\begin{tabular}{lcc}
\hline & $\begin{array}{c}\text { Dentists }(\%) \\
(\mathrm{n}=413)\end{array}$ & $\begin{array}{c}\text { DONs (\%) } \\
(\mathrm{n}=97)\end{array}$ \\
\hline $\begin{array}{l}\text { Interest of dentists in providing dental care for nursing } \\
\text { home residents }\end{array}$ & \\
Very/Extremely interested & 16.5 & 12.3 \\
Interested & 26.8 & 28.9 \\
Somewhat/Not interested & 56.7 & 58.8 \\
Were aware of change to dental & & \\
hygienist regulations & 39.9 & 24.0 \\
Were aware of ADA nursing home & & \\
dental scheme & 38.7 & 38.1 \\
Frequency of dental examination required for edentulous residents & 3.1 \\
When resident admitted & 7.6 & 19.6 \\
At a regular interval (3-24mths) & 44.3 & 37.1 \\
When admitted + regular interval & 38.8 & 28.9 \\
As required only & 6.8 & 11.3 \\
Exam by dentist not needed & 2.5 & 4.1 \\
Frequency of dental examination required for dentate residents \\
When resident admitted & 3.9 & 37.1 \\
At a regular interval (3-12mths) & 42.9 & 50.5 \\
When admitted + regular interval & 48.8 & 7.3 \\
As required only & 4.4 & 1.0 \\
Exam by dentist not needed & 0.0 & \\
\hline
\end{tabular}




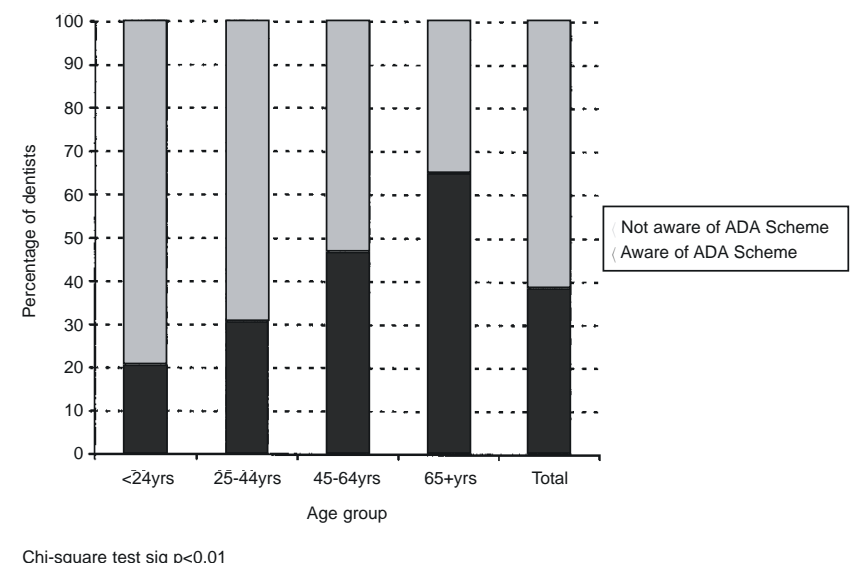

Fig. 2. Dentists' awareness of ADA Nursing Home Scheme by age group $(n=394)$.

allow for a more accurate comparison. Significant differences in standardised mean scores were evident for eight statements. These eight problems were:

- Four resident-related problems rated more frequently by DONs:

- residents' cognitive status

- residents' behavioural problems

- residents' financial constraints

- obtaining consent for residents' dental care; and

- Four nursing home/dental practice-related problems rated more frequently by dentists:

- dislike of providing regular oral hygiene care for residents by nursing home staff staff

- low priority given to dental care by nursing home

- increased time needed to provide dental treatment at nursing homes

- no suitable area available for dental treatment at nursing homes.

Of the remaining 11 problems, dentists and DONs both rated a group of five nursing home/dental practicerelated problems as the most frequently encountered:

- nursing home staffing and time constraints

- insufficient knowledge about dental care by nursing home staff

- transportation of residents to a dental practice/clinic

- preference of dentists to treat residents at their dental practice/clinic

- no portable dental equipment for use in nursing homes.

Dentists and DONs made many relevant and notable comments on the questionnaires. DONs emphasised

Table 4. Logistic regression - Dentists who provided care at nursing homes during the previous year.

\begin{tabular}{lc}
\hline & Odds Ratio \\
\hline Dentist characteristics & \\
Very/extremely interested in nursing home dentistry† $^{*}$ & 1.72 \\
Aware of ADA scheme* & 2.82 \\
Male† & 1.84 \\
\hline
\end{tabular}

*sig. $\mathrm{p}<0.01$

†sig. $\mathrm{p}<0.05$
Table 5. Dentists' and DONs' ratings of problems encountered with the organisation and provision of dental care for residents.

\begin{tabular}{|c|c|c|}
\hline & \multicolumn{2}{|c|}{$\begin{array}{c}\text { Standardised } \\
\text { mean scores } \\
(1=\text { always a problem; } \\
5=\text { never a problem })\end{array}$} \\
\hline & Dentists & DONs \\
\hline \multicolumn{3}{|c|}{ Resident-related problems rated more frequently by DONs } \\
\hline Cognitive status of residents* & 2.67 & 1.89 \\
\hline $\begin{array}{l}\text { Behavioural problems of } \\
\text { residents* }\end{array}$ & 2.78 & 2.08 \\
\hline Financial constraints of residents* & 2.76 & 2.29 \\
\hline $\begin{array}{l}\text { Obtaining consent for residents' } \\
\text { dental care* }\end{array}$ & 3.52 & 3.19 \\
\hline \multicolumn{3}{|c|}{$\begin{array}{l}\text { Nursing home/dental practice-related problems rated more } \\
\text { frequently by dentists }\end{array}$} \\
\hline $\begin{array}{l}\text { Dislike of providing regular oral } \\
\text { hygiene care for residents by } \\
\text { nursing home staff* }\end{array}$ & 2.52 & 3.36 \\
\hline $\begin{array}{l}\text { Low priority given to dental care } \\
\text { by nursing home staff* }\end{array}$ & 2.36 & 3.00 \\
\hline $\begin{array}{l}\text { Increased time needed to } \\
\text { provide dental treatment } \\
\text { at nursing homes* }\end{array}$ & 2.17 & 2.69 \\
\hline $\begin{array}{l}\text { No suitable area available for } \\
\text { dental treatment at } \\
\text { nursing homes* }\end{array}$ & 2.21 & 2.56 \\
\hline \multicolumn{3}{|c|}{$\begin{array}{l}\text { Nursing home/dental practice-related problems rated similarly by } \\
\text { dentists and DONs }\end{array}$} \\
\hline $\begin{array}{l}\text { Nursing home staffing and } \\
\text { time constraints } \\
\text { Insufficient knowledge about } \\
\text { dental care by nursing }\end{array}$ & 2.80 & 2.64 \\
\hline home staff & 2.44 & 2.63 \\
\hline $\begin{array}{l}\text { Transportation of residents to } \\
\text { a dental practice/clinic }\end{array}$ & 2.59 & 2.34 \\
\hline $\begin{array}{l}\text { Preference of dentists to treat } \\
\text { residents at their dental } \\
\text { practice/clinic }\end{array}$ & 2.53 & 2.46 \\
\hline $\begin{array}{l}\text { No portable dental equipment } \\
\text { for use in nursing homes }\end{array}$ & 2.02 & 2.11 \\
\hline
\end{tabular}

*t-test $\mathrm{p}<0.01$

their appreciation of the public dental domiciliary service. However, they indicated that waiting times were long and increasing, and that the service required more promotion in Adelaide nursing homes. 'We have a motivated and cooperative public domiciliary dentist, but with more elderly retaining teeth I don't think that the current service will be adequate in the future'. 'The public domiciliary dentists are extremely kind, gentle and caring for residents, but they have to be realistic about what they can cope with'.

DONs commented that private dentists were not prepared to come to nursing homes and were difficult to find. They commented that dentists needed specialised skills to care for residents with dementia and behavioural problems, especially kindness, compassion and patience. However, they felt that many dentists did not have these skills. 'Transport to facilities outside the nursing home is difficult because of residents' extreme frailty (mentally and physically)'. 'When you persuade private dentists to come they don't have equipment'. 'Dentists are unfamiliar with residents with dementia'. 'To care for debilitated patients takes specific skills - patience and kindness and ability to cope with confused/dementia patients; these patients can't be placed in a dental chair or be expected to sit'. 
Dentists commented that the increasing numbers of dentate nursing home residents was 'a potential time bomb if not addressed in a timely manner'. Their most frequent comments concerned the need for a coordinated, centralised plan/approach to dental care for nursing home residents, with regular dental examinations and government funding to all nursing homes. 'Nursing homes and government should be responsible for the cost of regular dental screenings and residents then pay only for treatment'. 'A certification of dental health and specific preventive care instructions should be issued upon admittance to the nursing home'.

Other issues frequently commented upon were:

- the difficult working conditions, and the lack of dental chairs in nursing homes;

- difficulty finding time for nursing home dentistry, the financial loss to dentists for nursing home dentistry, and the attitude that nursing home dentistry was 'community work';

- problems with the Pensioner Denture Scheme and Department of Veterans' Affairs rebates;

- problems instituting preventive care procedures in nursing homes, and the lack of knowledge among nursing home staff about preventive oral care;

- the need for more dental auxiliaries in nursing home dentistry; and

- unreasonable expectations from families, carers and residents.

\section{DISCUSSION}

Dental care provision for Adelaide nursing home residents, as reported by dentists and DONs, was low. Although many dentists did have links with one or more nursing homes, they were spending little time providing care for residents. When dentists did provide care, a clear pattern emerged with a distinct preference of dentists to treat residents at their dental practices/ clinics and not at nursing homes. In the half-hour to two hours per month spent on-site by the majority of dentists providing care, it seems probable that mainly screening and emergency type procedures were performed for a small number of residents.

These results revealed that both the public and private dental sectors were important sources of dental care for Adelaide nursing home residents. The favourable comments made by DONs supporting the public dental domiciliary service staff were encouraging. However, over 40 per cent of DONs had not used the service, and many had concerns about the domiciliary waiting times and care availability that need to be addressed. Increased administrative and financial support is needed to upgrade the public dental domiciliary service.

Sixty percent of DONs reported that a private dentist had not provided treatment at their nursing home for residents during the previous 12 months. DONs' comments highlighted their difficulties finding private dentists to come to nursing homes. DONs also recognised the concurrent trend for dentists' preference to treat residents at their dental practices/clinics and discussed the associated difficulties. An improved focus on dental treatment provision on-site at nursing homes is urgently required.

Of particular concern was the low number of hygienists reportedly involved in nursing home dentistry. In South Australia the dental profession recognised the need to involve hygienists in nursing home dentistry by changing the hygienist regulations in January 1997, permitting hygienists to work unsupervised in nursing homes to a dentists' prescribed treatment plan. ${ }^{18}$ However, the results of this study suggest that the regulatory change has had little impact on service provision.

Research conducted in nursing homes has emphasised the importance of ongoing, 'hands-on' educational interventions with nursing home staff to assist them to overcome the difficulties with providing residents' oral hygiene care, especially for residents with cognitive and behavioural problems. ${ }^{14}$ DONs in this study made many requests for increased educational support from dental professionals. South Australian dental professionals have the resources to provide this educational support. Dental hygienists can play an important role in the delivery of this educational assistance, and receive appropriate training in their South Australian course to do so. ${ }^{19}$ Also, in South Australia, ongoing support for qualified dental professionals interested in delivering educational programs to nursing homes is provided by The Alzheimer's Association of South Australia Dental Group. ${ }^{20}$

The importance and effectiveness of comprehensive undergraduate theoretical and clinical experiences in nursing home dentistry has been well researched..$^{21,22}$ These educational experiences improve both the attitudes of dentists toward nursing home dentistry and the dental services provided to residents. ${ }^{21,22}$ Traditionally, the education of Australian dentists in geriatric dentistry has not received a high profile, with very few clinical or theoretical courses available to undergraduates or postgraduates. ${ }^{23}$ Many dentists in this study commented on specific problems they had with nursing home dentistry and their lack of training in areas such as accessing the oral cavity of difficult residents, management of rampant caries, difficulties with denture construction, and physical limitations with the treatment of bed-ridden and wheelchair-bound residents. The results from this study illustrate the urgent need to upgrade Australian undergraduate, postgraduate and continuing dental education in geriatric dentistry for current and future dental professionals.

Recent changes in the Australian Aged Care System included the introduction of standards and guidelines for older adults residing in aged care facilities. ${ }^{24}$ In many industrialised countries the dental profession has been working toward the inclusion of regular dental examinations by dental professionals into nursing home protocols. ${ }^{25}$ In late 1997, a working group of Australian and international dental professionals, 
coordinated by the Federal ADA, assisted the Australian Commonwealth Government with advice concerning the standard and guidelines for 'oral and dental care'. This working group recommended that residents should have a dental examination conducted by a dental professional upon admission to an aged care facility and at regular intervals. However, this recommendation was not incorporated into the 1998 Australian Aged Care Standards. ${ }^{24}$ Policy makers must be aware of the concerns and attitudes of all professionals involved with the organisation and provision of dental care for residents, such as dentists and DONs. This study provided such information. The most frequent comments made by dentists expressed the need for a coordinated, centralised approach for nursing home dentistry, with financial support from government. Adelaide dentists and DONs supported both the examination of a resident by a dental professional when admitted to the nursing home, as well as on a regular basis.

The common perceptions held by dentists and DONs concerning problems related to care provision at nursing homes identified the inability of dental professionals to provide comprehensive clinical dental care on-site at nursing homes as the key issue to be addressed in nursing home dentistry in Adelaide. This resulted in the need for transportation of residents offsite to dental practices/clinics. DONs and dentists made many comments about this issue and identified several solutions, such as the hiring of portable dental equipment by dental professionals, the use of portable wheelchair headrests, and the improvement of clinical education for dental professionals in nursing home dentistry.

DONs' comments highlighted how residents' cognitive and behavioural problems often made the utilisation of off-site dental premises difficult, if not impossible. Dentists' inadequate awareness of and training about these resident-related problems complicated the situation even further.

Dentists were concerned with nursing home/dental practice-related problems and were frustrated with their perception of the low profile of dentistry in nursing homes. Dentists compromised themselves financially and made a loss to provide dentistry for nursing home residents. Time they spent at nursing homes and travelling between locations meant less time spent at their practices. Productivity was low when treating only one or two residents at nursing homes. Many residents could not afford to pay private dental fees so dentists charged them lower rates and classed this care as 'charitable or community work'. Without adequate financial reimbursement and a structured system for care provision, it is unlikely that nursing home dentistry can be a financially viable option for most public and private sector dental professionals.

DONs' responses provided clues to resolving dentists' frustrations with their perception that dental care was a low priority in nursing homes. As one DON commented: 'Dentists have a lack of insight and high expectations of nursing home residents and staff'. Residents' cognitive and behavioural problems dictated how residents' needs and care were prioritised in the nursing home. However, DONs commented frequently that dentists' lacked the skills to communicate with and treat cognitively impaired, behaviourally difficult and/or resistive residents. DONs highlighted how nursing home staffing and time constraints also interfered with dental care. DONs felt that dentists must understand and manage such problems better for their successful integration into the nursing home environment. DONs' suggestions to increase dentists' interest in nursing home dentistry included:

- the use of dental hygienists to provide preventive oral care and on-going staff educational programs;

- the appointment of a 'dental coordinator' or 'oral hygiene nursing assistant' at each nursing home;

- more clinical experiences in nursing homes for dentists and hygienists; and

- better working areas for dental treatment, supplemented with the donation of old dental chairs to nursing homes.

As this study questioned Adelaide DONs and dentists, the results cannot be directly extrapolated to other Australian cities or overseas situations. However, there were many similarities found among the Colorado, ${ }^{11}$ Canadian $^{13}$ and Adelaide study results concerning barriers/problems to dental care provision for nursing home residents. The Adelaide study findings may assist providers of dental care and nursing homes to better understand and improve dental care provision for their residents. This study also provides a study framework for replication with dental and nursing professionals in other areas of Australia.

Several areas of interest not included in this Adelaide study questionnaire to be addressed in future studies are:

- possible arrangements/contracts between dentists and nursing homes;

- types of dental services provided at dental practices versus at nursing homes;

- types of portable dental equipment used in nursing homes;

- actual numbers of residents cared for at each nursing home; and

- the influence and use of the nursing home dental standard and guidelines.

The questionnaire was not sent to dental technicians or dental hygienists. Quantification of their involvement in service provision for nursing home residents and their attitudes toward nursing home dentistry is needed.

\section{CONCLUSIONS}

Dental care provision for residents of Adelaide nursing homes was reported, by both dentists and directors of nursing, to be low. There was a distinct pattern of service provision evident in which dentists 
preferred to treat residents at their dental practices and not on-site at nursing homes. The reported use of dental hygienists in nursing homes was minimal.

The majority of Adelaide dentists felt they had inadequate training in nursing home dentistry and had low levels of interest in nursing home dentistry. There was very little assistance given to nursing homes by dental professionals with staff education concerning residents' oral care. Most dentists and directors of nursing were unaware of the Australian Dental Association (SA Branch) Nursing Home Scheme and changes to the dental hygienist regulation. The majority of dentists and directors of nursing indicated that dental examinations conducted by a dentist were required for residents (especially dentate residents) upon admission to the nursing home and at regular intervals. Many dentists and DONs commented that a centrally coordinated and financed approach was needed for nursing home dentistry involving government support.

Dentists and DONs held several common and many varying perceptions of the problems they encountered with the organisation and provision of dental care for nursing home residents. Both dentists and directors of nursing identified the problems associated with the inability of Adelaide dentists to provide dental care onsite at nursing homes. The lack of portable dental equipment available and lack of suitable areas for dental treatment in nursing homes, resulted in the preference of dentists to provide treatment at their practices/clinics and in the need to transport residents to access dental care. Dentists further identified nursing home/dental practice-related problems such as increased time needed for nursing home dentistry, low financial reimbursement, nursing home staff's dislike of providing regular oral hygiene care for residents, and the low priority given to dentistry in nursing homes. Directors of nursing further identified resident-related problems such as cognitive status, behavioural problems, financial status and obtaining consent for dental care.

\section{ACKNOWLEDGEMENTS}

This study was funded by the Australian Dental Association South Australian Branch and the AIHW Dental Statistics and Research Unit, The University of Adelaide. Many thanks to all the dentists and directors of nursing who kindly participated in this study.

\section{REFERENCES}

1. Vowles NJ, Watson BI, Dahl BJ. The needs of the homebound and institutionalised in South Australia, 1977. Aust Dent J 1979;24:114-120.

2. Crack DJ, McDougall WA, Spencer AJ. Dental needs of the handicapped and homebound. The Royal Dental Hospital of Melbourne, 1980.

3. Walker BN. Dental survey of nursing home residents in South Australia. Aust Dent J 1984;29:305-307.

4. Stockwell AJ. Survey of the oral health needs of institutionalised elderly patients in Western Australia. Comm Dent Oral Epid 1987;15:273-276.
5. Homan BT, Lam B, Larsen RG. The oral needs and demands of a geriatric population at Mt Olivet, Brisbane, 1986. Aust Dent J 1988;33:424-432.

6. Berkey DB, Ettinger RL, Meskin LH. Oral health care variables affecting the institutionalised and homebound individual: a review and analysis of the literature 1970-1989. Iowa Geriatric Education Center. Monograph Series, Number 3, 1990.

7. Thomson WM, Harvey Brown R, Williams SM. Dental status and treatment needs of a New Zealand institutionalised elderly population. NZ Dent J 1992;87:119-123.

8. Dolan TA, Atchison KA. Implications of access, utilisation and need for oral care by the non-institutionalised and institutionalised elderly in the dental delivery system. J Dent Educ 1993;57:876-887.

9. Chalmers JM, Hodge C, Fuss J, Spencer AJ, Carter KD. The Adelaide Dental Study of Nursing Homes. Baseline Data Collection Report 1998. AIHW Dental Statistics and Research Unit, The University of Adelaide. 1999.

10. MacEntee MI, Weiss R, Waxler-Morrison NE, Morrison BJ. Factors influencing oral health in long term care facilities. Comm Dent Oral Epid 1987;15:314-316.

11. Berkey DB, Call RL, Gordon SR, Berkey KG. Barriers influencing dental care in long-term care facilities. Gerodontics 1988;4:315-319.

12. MacEntee MI, Weiss RT, Waxler-Morrison NE, Morrison BJ. Opinions of dentists on the treatment of elderly patients in longterm care facilities. J Public Health Dent 1992;52:239-244.

13. Weiss RT, Morrison BJ, MacEntee MI, Waxler-Morrison NE. The influence of social, economic, and professional considerations on services offered by dentists to long-term care residents. J Public Health Dent 1993;53:70-75.

14. Chalmers JM, Levy SM, Buckwalter KC, Ettinger RL, Kambhu PP. Factors influencing nurses' aides' provision of oral care for nursing facility residents. Spec Care Dent 1996;16:71-79.

15. MacEntee MI, Thorne S, Kazanjian A. Conflicting priorities: oral health in long-term care. Spec Care Dent 1999;19:164-172.

16. Pyle MA, Nelson S, Sawyer DR. Nursing assistants' opinions of oral health care provision. Spec Care Dent 1999;19:112-117.

17. Johnson TE, Lange BM. Preferences for and influences on oral health prevention: perceptions of directors of nursing. Spec Care Dent 1999;19:173-180.

18. SA Dental Board, 1997. Restrictions and conditions on the provision of dental treatment by dental hygienists. Amended Regulation 12 Under SA Dentists ACT - Gazetted 1/97.

19. Chalmers JM. 1998a. Dental hygiene - geriatric dentistry module. School of Dental Hygiene, Gilles Pains TAFE, 1998

20. Chalmers JM, Gryst M, Jolly M. Alzheimer's Association of South Australia. Dental Group Handbook, 1997.

21. Ettinger RL, Beck JD. Geriatric dental curriculum and the needs of the elderly. Spec Care Dent 1984;4:207-213.

22. Ettinger RL. The status of geriatric dental education in the USA. In Geriatric and Special Care Dentistry Workshop - Proceedings. AIHW Dental Statistics and Research Unit, The University of Adelaide, 1997.

23. Chalmers JM, Fuss J, Gryst M. 1998b. Special care dentistry curriculum review. Department of Dentistry, The University of Adelaide. 1998.

24. Commonwealth Department of Health and Family Services, 1998. Standards for Aged Care Facilities. Standard 2: Health and Personal Care. Standard 2.15 Oral and Dental Care. November 1998. http://www.health.gov.au/acc/standard/facility/std2.htm

25. Special Care in Dentistry 1996;164. Issue dedicated to Proceedings, white papers, and recommendations from the consensus conference on developing practice guidelines for institutionalised older dental patients.

Address for correspondence/reprints: Dr Jane Chalmers AIHW Dental Statistics and Research Unit The University of Adelaide Adelaide, South Australia 5005 